\title{
Diagnostic Value of Bronchoalveolar Lavage
}

\author{
Acharya $S^{1}$, Yogi $S^{2}$
}

\begin{abstract}
Introduction: Bronchoalveolar lavage has a high diagnostic utility for cytology and bacteriology. It can be done as an outpatient procedure for both immunocompetent and immunocompromised patient. Material obtained by bronchoalveolar lavage can give a definite diagnosis in conditions such as infections and malignancies. Aim: The aim of this study was to assess the diagnostic value of bronchoalveolar lavage (BAL) in cases which underwent routine bronchoscopy for evaluation of lung disease. Materials and methods: This is a hospital based descriptive study done from $16^{\text {th }}$ June 2016 to $15^{\text {th }}$ June2017. One hundred twenty bronchoalveolar lavage (BAL) cases were analyzed for differential count, cytological evaluation and bacteriological examination. All cases were included which were sent as BAL specimen to the laboratory department. Bronchoscopy was done as an outpatient procedure and lavage fluid obtained was analyzed. Result: Out of 120 cases, 69 were male and 51 were female. The age ranged from 10 to 80 years. Among 120 cases, eight ( $n=6.66 \%)$ cases were unsatisfactory, twelve $(n=10 \%)$ cases were of tuberculosis, one $(n=0.83 \%)$ case was of fungal infection, two $(n=1.67 \%)$ cases were of malignancy, ninety one $(n=75.84 \%)$ cases were of small airway infection and six cases were satisfactory but with no diagnostic value. $(n=5 \%)$. Conclusion: Bronchoalveolar lavage is valuable in diagnosis of tuberculosis, infections and malignancies.
\end{abstract}

Key words: Bronchoalveolar lavage (BAL), immunocompetent, immunocompromised

\section{INTRODUCTION}

Bronchoalveolar lavage (BAL) is a saline fluid obtained through outpatient procedure in which fiberoptic bronchoscope is inserted through nose or mouth in selected bronchopulmonary segment. Minimum of $100 \mathrm{ml}$ and maximum of $300 \mathrm{ml} \mathrm{normal}$ saline is installed into particular segment and reaspirated by bronchoscope. A minimal volume of $5 \mathrm{ml}$ of a pooled BAL sample is needed for cellular analysis. The optimal volume is 10 to $20 \mathrm{ml}^{1}$.

Bronchoalveolar lavage cellular differential counts with greater than $15 \%$ lymphocytes are labeled as lymphocytic cellular pattern, greater than 3\% neutrophils as neutrophilic cellular pattern, greater than $1 \%$ eosinophils as eosinophilic cellular pattern, and greater than $0.5 \%$ mast cells represent as mastocytosis ${ }^{1}$.

Cytologic examination in bronchoalveolar lavage has been used to identify malignancy. Criteria to detect for malignancy in bronchoalveolar lavage fluid samples are similar as in other procedure ${ }^{2}$. In bacteriological study, bronchoalveolar lavage (BAL) is the best diagnostic material even in sputum smear negative cases for the diagnosis of pulmonary tuberculosis ${ }^{3}$.

1. Dr. Subarna Acharya

2. Dr. Subash Yogi

Address for correspondence:

Dr. Subarna Acharya

Department of Pathology

Nepalgunj Medical College Teaching Hospital

Nepalgunj, Banke, Nepal

Email: subarnaacharya40@gmail.com
The number of studies on BAL in Nepali literature is few. This study is done to highlight the diagnostic value of BAL material in making a definite diagnosis. BAL material has a very important role in diagnosis of infections and malignancies. It is a relatively safe procedure and is well tolerated.

\section{MATERIAL AND METHOD}

Bronchoalveolar lavage was obtained in one hundred and twenty cases over a period of one year. Procedure was done at medicine department as outpatient procedure and lavage fluid obtained was analyzed. Differential count was done on airdried slide stained by Leishman stain. Routine Giemsa and PAP stains were done for cytology screening. Stain for acid fast bacilli (AFB) was done on all BAL samples.

\section{Inclusion criteria:}

All cases which were sent as BAL specimen to the laboratory department within study period were included. Adequacy of samples was assessed based on definite criteria. Chamberlain et al. criteria were used to categorize sample as unsatisfactory for evaluation under microscope ${ }^{4}$.

The criteria are -

1. Paucity of alveolar macrophages $<10 / 10 \mathrm{hpfs}$.

2. Extensive epithelial cells.

3. Mucopurulent exudates.

4. Numerous red blood cells.

5. Degenerating changes.

\section{RESULT}

Bronchoalveolar lavage was done in 120 cases. Age of patients ranged from 10 years to 80 years; 69 were males and 51 were females. Out of the 120 cases, eight were unsatisfactory for 
evaluation, fungal infection in one case [figure 1], tuberculosis in twelve cases, malignancy in two cases [figure 2], small airway infection in ninety one cases and satisfactory but with no diagnostic value in six cases. [Table I].

In cellular differential count, neutrophilic cellular pattern was seen in 68 cases, lymphocytic cellular pattern in two cases and absence of any cellular pattern in 50 cases including eight unsatisfactory cases. [Table II].

\begin{tabular}{|l|c|}
\hline Diagnosis & $\begin{array}{c}\text { Number } \\
\text { of cases }\end{array}$ \\
\hline Unsatisfactory & 08 \\
\hline Pulmonary Tuberculosis & 12 \\
\hline Fungal infection & 01 \\
\hline Malignancy & 02 \\
\hline Small airway infection & 91 \\
\hline Satisfactory but with no diagnostic value & 06 \\
\hline
\end{tabular}

Table I: Distribution of cases based on cytological diagnosis

\begin{tabular}{|l|c|}
\hline Diagnosis & $\begin{array}{c}\text { Number } \\
\text { of cases }\end{array}$ \\
\hline Neutrophilic cellular pattern & 68 \\
\hline Lymphocytic cellular pattern & 02 \\
\hline Absence of any cellular pattern & 50 \\
\hline
\end{tabular}

Table II: Distribution of cases based on cellular differential count

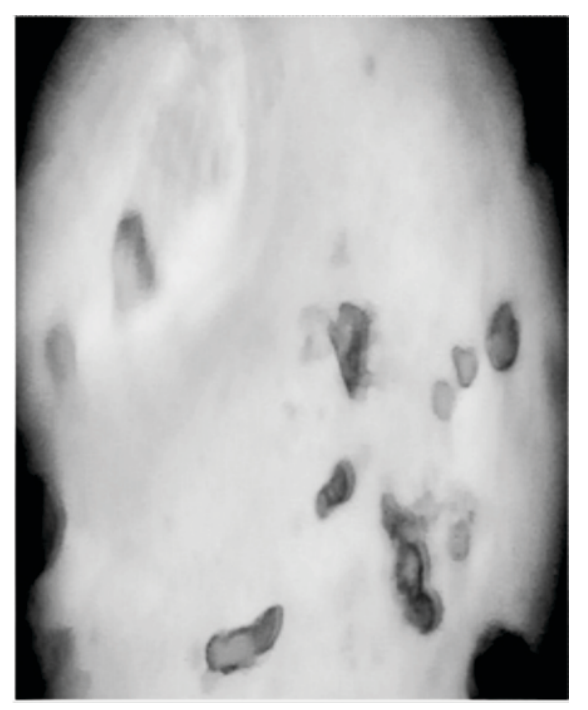

Figure 1: Fungal spores (Periodic acid Schiff stain PAS, $\mathrm{x} 1000)$

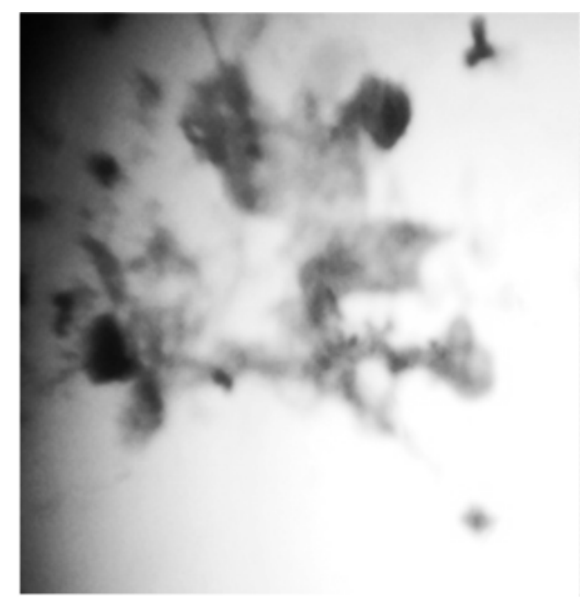

Figure 2: Singly dispersed malignant squamous cells (Pap, $\times$ 400)

\section{DISCUSSION}

In this study, attempt to assess the diagnostic value of BAL by cytology; bacteriology and cellular differential count have been made. It is easier and better investigative tool compared to other techniques like aspiration cytology and needle biopsy ${ }^{5}$. Result can be obtained in lesser time as compared to histopathology which is helpful in treatment modalities ${ }^{5}$. It helps to diagnose tuberculosis in sputum negative tuberculosis ${ }^{3}$. In this study, cases which were positive with acid fast bacilli stain and diagnosed as tuberculosis in BAL have shown neutrophilic cellular pattern only.

Eum SY et al. ${ }^{6}$ have also found similar findings in BAL study as predominance of neutrophilic cellular pattern in cases of tuberculosis [Table III].

\begin{tabular}{|l|c|}
\hline Diagnosis in BAL & Cellular pattern \\
\hline Pulmonary tuberculosis & Neutrophilic cellular \\
(Present study) & pattern \\
\hline Pulmonary Tuberculosis & Neutrophilic cellular \\
(Eum SY et al) $^{6}$ & pattern \\
\hline
\end{tabular}

Table III: Comparison for predominance of neutrophilic cellular pattern in pulmonary tuberculosis diagnosed in bronchoalveolar lavage with other study

Lymphocytic cellular pattern more than $25 \%$ suggests sarcoidosis, cellular nonspecific interstitial pneumonia, drug reaction, lymphoid interstitial pneumonia, cryptogenic organizing pneumonia, or lymphoma. Neutrophilic cellular pattern more than $50 \%$ supports acute lung injury, infection, aspiration pneumonia, or suppurative infection. Eosinophilic cellular pattern more than $25 \%$ is diagnostic of acute or chronic eosinophilic pneumonia ${ }^{1}$. In this study a case was diagnosed as fungal infection in alcohol fixed smears and was confirmed by Periodic acid Schiff (PAS) stain. Knox KS and Meinke L ${ }^{7}$ have also 
concluded that bronchoalveolar lavage is a supplementary diagnostic tool to culture for diagnosis of pulmonary and disseminated fungal infections.

Two cases were diagnosed as malignancy in present study. Levy et $a .^{8}$ concluded that findings of malignancy in BAL was superior (66\%) as compared to washings (57\%), brushings (40\%) and transbronchial biopsy (44\%). BAL has been used for therapeutic applications as well. Whole lung lavage is a treatment for pulmonary alveolar proteinosis ${ }^{9}$.

One of the major limitations of bronchoalveolar lavage is classification of interstitial lung diseases. However, categorization of interstitial lung disease has fewer roles in therapeutic management $^{10}$.

\section{CONCLUSION}

Bronchoalveolar lavage has a high yield in diagnosis of tuberculosis, fungal infection and malignancies. Definite diagnosis outnumbered the undiagnosed cases so we conclude that bronchoalveolar lavage should be used as routine diagnostic tool for lung diseases. It can be used for culture, ancillary techniques and research purpose.

\section{REFERENCES}

1. Meyer KC, Raghu G, Baughman RP, Brown KK, Costabel U, du Bois RM, et al. An official American Thoracic society clinical practical guideline: The clinical utility of bronchoalveolar lavage cellular analysis in interstitial lung disease. Am J Respir Critic Care Med. 2012;185:1004-14.

2. Rennard SI. Bronchoalveolar lavage in the diagnosis of cancer. Lung. 1990;168:1035-40.

3. Nikbakhsh N, Bayani M, Siadati S. The value of Bronchoalveolar Lavage in the Diagnosis of Sputum Smear - Negative Pulmonary Tuberculosis. Iran J Pathol. 2015;10(1):35-40.

4. Chamberlain DW, Braude AC, Rebuck AS. A critical evaluation of bronchoalveolar lavage. Criteria for identifying unsatisfactory specimens. Acta Cytol. 1987;31:599-605.

5. Radha S, Afroz T, Prasad S, Ravindra N. Diagnostic utility of bronchoalveolar lavage. J Cytol. 2014;31(3):136-8

6. Eum SY, Kong JH, Hong MS, Lee YJ et al. Neutrophils are the predominant infected phagocytic cells in the active pulmonary TB. Chest. 2010;137(1):122-8.

7. Knox KS, Meinke L. Role of bronchoalveolar lavage diagnostics in fungal infections.

8. Levy $\mathrm{H}$, Horak DA, Lewis MI. The value of bronchial washings and bronchoalveolar lavage in the diagnosis of lymphangitic carcinomatosis. Chest. 1988;94:1028-30.

9. Michaud G, Reddy C, Ernst A. Whole - lung lavage for pulmonary alveolar proteinosis. Chest. 2009;136(6):1678-81.

10. Kebbe J, Abdo T. Interstitial lung disease: the diagnostic role of bronchoscopy. J Thorac Dis. 2017;9(10):996-1010. 